\title{
The Thermal Inertia Characteristics of the System Ocean-Atmosphere
}

\author{
Habibullo I. Abdussamatov, Sergey I. Khankov, Yevgeniy V. Lapovok \\ Space Research of the Sun Sector, Pulkovo Observatory, St. Petersburg, Russia \\ Email: abduss@gao.spb.ru,khankovsi@gmail.com
}

Received August 7, 2012; revised September 6, 2012; accepted October 8, 2012

\begin{abstract}
To estimate the time delay between the planetary temperature change and the change of the incoming solar radiation fraction absorbed by the ocean and the atmosphere, the analytical energy balance model is presented. The model generalization allows of using averaged data for model parameterization. Using the model, the time delay is investigated on four model cases of absorbed radiation change. The interconnections among the time delay, the planetary thermal inertia and the ocean active layer depth are established.
\end{abstract}

Keywords: Thermal Inertia; Planetary Temperature; Total Solar Irradiance; System Ocean-Atmosphere

\section{Introduction}

Theoretical studies of the Earth's temperature change related to the change of the solar incoming absorbed by the Earth are based on model development with parameterization based on empirical data. Main approaches to model development are general circulation models (GCMs), statistical dynamical models (SDMs) and energy balance models (EBMs).

EBMs have been developed extensively after Budyko [1] and Sellers [2] published their models. Models of this type are relatively simple in comparison with GSMs and place more emphasis upon obtaining strong theoretical and analytical results in comparison with SDMs. Commonly for EBM, the model surface temperature is an unknown function of time obtaining from the one-dimensional equation, whereas zonal and seasonal variations of key climate parameters are taken into account through empirical data. The paper [3] contains a reexamination of EBMs developed before year 1981, and the papers [4-7] are samples of the results of modern EBM development.

The important attribute of the models described above is their attempt to include the zonal and seasonal variations of the outgoing infrared flux, the incoming solar flux and the space-dependent heat capacity. This model presents the energy balance model with two important features. First, our model is generalized and based on the specific heat capacity for the ocean and the atmosphere per unit surface $\left[\mathrm{m}^{2}\right]$ averaged over the Earth's surface and on the annual averaged solar irradiation. Second, the Earth is considered as a system of the ocean and the at- mosphere.

This approach makes it possible to research the dynamic of the transition of the system ocean-atmosphere from one quasi-equilibrium state to another for model cases of the absorbed solar flux change. In addition, this approach makes it possible to reduce the term "the climate sensitivity" to the "thermal inertia" determined mainly by the ocean heat capacity.

Our study is aimed at determination of the time lag $\tau$ between the increment of the global planetary thermodynamic temperature, $\vartheta=T_{p}-T_{p o}$, (here $T_{p}$ and $T_{p o}$ are the planetary temperature and its initial value, respectively) and the increment of the specific power of thermal emission, $\Delta Q$, in the ocean and the atmosphere, caused by fluctuations of the total solar irradiance and/or Bond albedo. Our investigations have been performed for typical model cases of the temporal changes of the increment $\Delta Q$ which simplifies determination of the factors decelerating the process of transition into a new thermal state.

\section{Mathematical Modeling}

To investigate the planetary thermal inertia and the time lag, it is necessary to investigate the Earth's heat transient state taking into account the absorbed solar irradiation and the outgoing infrared flux.

In the previous paper [8] we obtained the non-steady equation of the planetary heat balance. Given the increment of the planetary absorbed solar irradiation and the related planetary temperature increment $\vartheta$, the equation is reconsidered in form 


$$
\begin{aligned}
& t_{p} \frac{\mathrm{d} \Theta}{\mathrm{d} \tau}+(1+\Theta)^{4}-1=\Delta \Theta_{o} f(\tau) ; \\
& t_{p}=\frac{c}{a} ; \quad \Theta=\frac{\vartheta}{T_{p o}} ; \\
& \Delta \Theta_{o}=\frac{\Delta Q}{\alpha T_{p o}} ; \quad \alpha=\frac{q}{T_{p o}}=a \sigma T_{p o}^{3}
\end{aligned}
$$

where $t_{p}$ is the constant of thermal inertia of the planet for the wide range of temperature change; $c$ is the surface density of the total thermal capacity of the system ocean-atmosphere; $\alpha$ is the heat transfer coefficient for the case of the planet radiation into open space; $\Theta$ is the relative increment of the planetary temperature; $\sigma$ is the Stefan-Boltzmann constant; $q$ is the specific power of thermal radiation of the planet into the space; $a$ is the dimensionless coefficient determined by the radiation characteristics of the Earth surface and atmosphere in the IR range [8,9]; $f(\tau)$ is the function describing the law of temporal variations of the increment $\Delta Q$ (decrease or increase of the planetary temperature is determined by the long-term variations of the sign of $\Delta Q$ ).

In case of small increments of the planetary temperature $\vartheta$ the value $\Theta<<1$ and the non-stationary equation of the thermal balance of the planet [9] can be written in the form admitting analytical solutions for important typical functions $f(\tau)$.

$$
\begin{aligned}
& t \frac{\mathrm{d} \vartheta}{\mathrm{d} \tau}+\vartheta=\vartheta_{m} f(\tau) ; \\
& t=\frac{c}{4 \alpha} ; \quad \vartheta_{m}=\frac{\Delta Q}{4 \alpha}
\end{aligned}
$$

where $t$ is the constant of thermal inertia of the planet;

In order to solve this equation, it is necessary to explicitly specify a function $f(\tau)$, which should correspond to possibly real processes as well as allow analytical description thus making possible to get analytical solution. If one uses dimensionless solution in the form $F(\tau)=$ $\vartheta / \vartheta_{\mathrm{m}}$, the problem comes down to determining the time lag between the functions $F(\tau)$ and $f(\tau)$. The value of $\Delta \tau$ can be derived from the expression:

$$
f(\tau)=F(\tau+\Delta \tau)=F(\tau, t=o)
$$

We have examined four variants of the function $f(\tau)$ and corresponding $F(\tau)$ obtained by solving the Equation (2) with initial condition $\vartheta(\tau=0)=0$.

1) A hypothetical case of jump discontinuity of $\Delta Q$ :

$$
F(\tau)=1-\exp \left(-\frac{\tau}{t}\right) \text { with } f(\tau)=1
$$

This case corresponds to the catastrophe like rapid change of Bond's albedo or atmospheric transparency.

2) A hypothetical case of linear dependence of the absorbed power, when $f(t)=t / t_{\mathrm{o}}$. Here $t_{\mathrm{o}}$ is the heat- ing/cooling time, $0 \leq \tau \leq t_{\mathrm{o}}$.

$$
F(\tau)=\frac{\tau}{t_{o}}(1-\phi) ; \quad \phi=\frac{1-\exp (-\bar{\tau})}{\bar{\tau}} ; \quad \bar{\tau}=\frac{\tau}{t}
$$

This case correspond to the possible scenario of the Bond's albedo change as a result of the ice cover change or the cloud cover change.

3) A hypothetical case of exponential change of the absorbed power with time, $f(\tau)=1-\exp \left[-k\left(\tau / t_{o}\right)\right]$ :

$$
\begin{aligned}
& F(\tau)=1-M_{1} \exp \left(-k \frac{\tau}{t_{o}}\right)+M_{2} \exp \left(-\frac{\tau}{t}\right) ; \\
& M_{1}=\frac{t_{o}}{t_{o}-k t} ; \quad M_{2}=\frac{k t}{t_{o}-k t},
\end{aligned}
$$

where $k$ is the coefficient determining the gradient of $\Delta Q$.

This case corresponds to Bond's albedo change during the process of the system ocean-atmosphere transition from one quasi-equilibrium state to another.

4) The case of periodic variations of the solar irradiance with, for example, bicentennial period can be described by the harmonic function

$$
f(\tau)=\sin \left(2 \pi \frac{\tau}{t_{o}}\right)
$$

The function $F(\tau)$ derived from solving the Equation (1) has a form:

$$
\begin{aligned}
& F(\tau)=\left(1+b^{2}\right)^{-1}\left\{\sin \left(2 \pi \tau / t_{o}\right)-b \cdot g\right\} \\
& g=\cos \left(2 \pi \tau / t_{o}\right)-\exp (-\tau / t) \\
& b=2 \pi \frac{t}{t_{o}}
\end{aligned}
$$

In the quasi-steady state regime, when the duration of the process is long enough (as for the system Sun-Earth) the latter term is close to zero. Then one can derive the time lag between the functions $F(\tau+\Delta \tau)$ and $f(\tau)$ from the distance between their nearest nodes equating both functions $(f$ and $F)$ to zero.

$\Delta \tau$ can be evaluated using the expression:

$$
\Delta \tau=\frac{t_{o}}{2 \pi} \operatorname{arctg} b
$$

To estimate the value of $t$ we have adopted the values of initial parameters which are realized nowadays and determined in our previous papers [8-10]. Then the value of $t$ can be defined according to the following dependence on the depth of the ocean's active layer:

$$
t=0.095(1+0.42 H)
$$

where $t$ is given in years, $H$-in meters.

It is worthwhile to estimate the minimum value of the depth of the ocean's active layer assuming an absence of 
vertical convection, that is when the process of heat transfer is determined only by the heat conductivity of the sea-water. Applying the second Fourier law one can evaluate the depth of the layer in which the temperature increment from the ocean's surface (corresponding to vertical coordinate $z=0$ ) down to the depth $z$ changes from от $\theta_{\mathrm{o}}$ to $\theta_{\mathrm{z}}$. This depth is determined according to expression:

$$
z=\sqrt{\frac{a t_{0}}{\pi}} \cdot \ln (K) ; \quad K=\frac{\theta_{o}}{\theta_{z}}
$$

where $a$ is the temperature transfer coefficient of the sea-water, $K$ is a relative decrease of the temperature increment at the depth $z$ with respect to the surface value.

Adopting the values $K=10$ and $K=100$ for the bicentennial cycle we get $z=300 \mathrm{~m}$ and $z=600 \mathrm{~m}$, respectively. The influence of convection can be accounted for using the convection coefficient, however its global value is difficult to determine. It is known only that it is greater than unity and is multiplied by the temperature transfer coefficient in the Equation (11), and therefore, the real value of the depth of the ocean's active layer exceeds the values mentioned above $(H>z)$. It is necessary to note that the notion of the depth of the ocean's active layer is rather conventional since the value of $\mathrm{H}$ (which is proportional to $z$ ) depends on an arbitrary taken value of $K$. It is important to emphasize that both the results of calculations for the case of harmonic fluctuations of the temperature of the ocean's surface layer (Figure 1) and estimations on the basis of formula (11) with the adequate choice of the criterion for attenuation of temperature variations $K$, the time lag ranges from 15 to 20 years.

\section{Calculation Results and Discussions}

In case of abrupt change of $\Delta Q$ the transition time between two steady states is totally and unambiguously determined by the constant of thermal inertia, $\tau \approx(3 / 4) t$, with an exact value being determined by a given error.

In case of linear and exponential changes of absorbed energy the lag of the temperature increment increases with time. On the final stage $\Delta \tau=\mathrm{t}$ for the linear law, and $\Delta \tau=\tau / M_{2}$ for the exponential law.

Dependences $t(H)$, calculated according to expression (10), and $\Delta \tau(H)$ determined from (9) are presented in Figure 1 Our calculations have shown that in case of harmonic fluctuations the time lag $\Delta \tau$ is smaller than the constant of thermal inertia. This can be explained from the physical point of view analyzing expressions (9) and (10). Expression (10) allows concluding that in case of infinite growth of $H$ (in a purely hypothetical case) the value of thermal inertia should increase infinitely almost proportionally to the value of $H$.
However, with the growth of $\mathrm{H}$ and, correspondingly, $t$, the value of $b$ which is proportional to $t$ (see Equation (8)) increases infinitely as well. But $\operatorname{arctg}(b)$ is limited to $\pi / 2$. Hence the value of $\Delta \tau$ in the expression (9) is limited to $t_{o} / 4$. For the bicentennial period and all real and even hypothetical values of $\mathrm{H}$ the time lag cannot exceed 50 years, and the function $\Delta \tau(H)$ is growing with saturation (line 2 in Figure 1).

\section{Conclusions}

The formulation of the equation of the Earth energy balance for the case of small increments of the solar power absorbed by the ocean and the atmosphere leads to the analytical formulas making it possible to calculate the planetary temperature change in time.

Using the obtained solutions, the time lag between the planetary temperature change and the planetary absorbed power change was investigated for four cases of the change of power absorbed by the ocean and the atmosphere in time.

The thermal inertia constant is defined for the wide range of the temperature change which corresponds to the Equation (1). Also, the thermal inertia constant is defined for the small increment of the absorbed power and the temperature, respectively, which corresponds to the Equation (2). The calculation results for the non-linear Equation (1) and the linearized Equation (2) are in agreement with $\pm 10 K$ accuracy. The linearization error of the Equation (2) raises with the increase of the temperature variations.

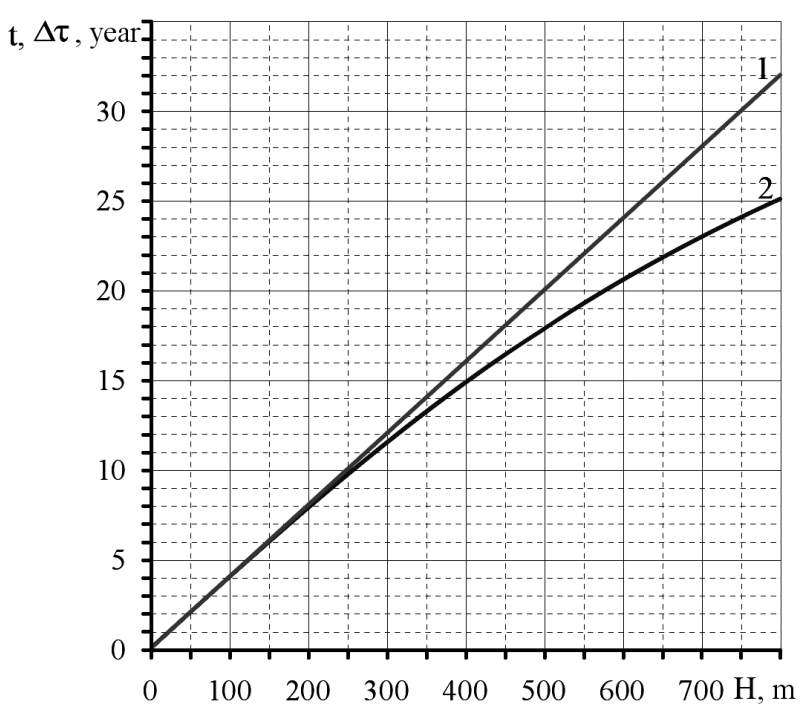

Figure 1. Dependence of the thermal inertia constant $t$ calculated using the expression (9), line (1), and of the time lag $\Delta \tau$ calculated using the expression (8), line (2), on the depth of the ocean's active layer $H$ for the case of harmonic bicentennial variations of the absorbed thermal energy defined by expression (6). 
The time lag dependence on the thermal inertia value is estimated for each case of the $f(\tau)$ where the thermal inertia is defined by the ocean active layer depth (10).

The formula (11) is offered to estimate the ocean active layer depth. The formula (11) leads to the conclusion that in case of harmonic form of $f(\tau)$ the time lag would range from 15 to 20 years.

Since the real bicentennial cyclic variations of the total solar irradiance are not purely harmonic and contain dips and glitches of 11-year cycles, the real time lag is determined by more complicated dependences. The changes of $\Delta Q$ can be caused also by long-term variations of the Bond albedo. The character of its variations is poorly understood so far, therefore both the linear and exponential presentations of the function $f(\tau)$ can be used for approximation of real variations during certain time intervals.

\section{REFERENCES}

[1] M. I. Budyko, "The Effect of Solar Radiation Variations on the Climate of Earth," Tellus, Vol. 21, No. 5, 1969, pp. 611-619. doi:10.3402/2Ftellusa.v21i5.10109

[2] W. D. Sellers, "A Climate Model Based on the Energy Balance of the Earth-Atmosphere System," Journal of Applied Meteorology and Climatology, Vol. 8, 1969, pp. 392-400. doi: $10.1175 / 2 \mathrm{~F} 1520-0450 / 281969 \% 29008 \% 3 \mathrm{C} 0392 \% 3 \mathrm{~A}$ AGCMBO $\% 3 \mathrm{E} 2.0 . \mathrm{CO} \% 3 \mathrm{~B} 2$

[3] G. R. North, R. F. Cahalan and J. A. Coakley Jr., "Energy Balance Climate Models," Reviews of Geophysics, Vol. 19, No. 1, 1981, pp. 91-121.

\section{doi:10.1029/2FRG019i001p00091}

[4] C. E. Graves, W. Lee and G. R. North, "New Parameterization and Sensetivities for Simple Climate Models," Journal of Geophysical Research, Vol. 98, No. D3, 1993, pp. 5025-5036. doi:10.1029/2F92JD02666

[5] J. I. Diaz, G. Hetzer and L. Tello, "An Energy Balance Climate Model with Hysteresis," Nonlinear Analysis, Vol. 64, No. 9, 2006, pp. 2053-2074. doi:10.1016/2Fj.na.2005.07.038

[6] A. V. Karnaukhov, "Role of the Biosphere in the Formation of The Earth's Climate: The Greenhouse Catastrophe," Biophysics, Vol. 46, No. 6, 2001, pp. 1078-1088.

[7] B. G. Sherstyukov, "Thermal Inertia of the Ocean and the Green-House Effect in the Modern Climate Variations," Meteorology and Hydrology, No. 7, 2006, pp. 66-72.

[8] H. I. Abdussamatov, A. Bogoyavlenskii, Y. V. Lapovok and S. I. Khankov, "The Influence of the Atmospheric Parameters Determining the Transparency of Solar and Earth's Radiation on the Climate," Proceedings of the All-Russian Annual Conference "Solar and Solar-Terrestrial Physics -2010”, Saint-Petersburg, 2010, pp. 7-10.

[9] H. I. Abdussamatov, A. I. Bogoyavlenskii, Y.V. Lapovok and S. I. Khankov, "Modeling of the Earth's Planetary Heat Balance with Electrical Circuit Analogy," Journal of Electromagnetic Analysis and Applications, Vol. 2, No. 3, 2010, pp. 133-138. doi:10.4236/jemaa.2010.23020

[10] H. I. Abdussamatov, A. I. Bogoyavlenskii, Y. V. Lapovok and S. I. Khankov, "The Influence of the Atmospheric Transmission for the Solar Radiation and Earth's Surface Radiation on the Earth's Climate," Journal of Geographic Information System, Vol. 2, No. 10, 2010, pp. 194-200. doi:10.4236/jgis.2010.24027 